\title{
Global Sensitivity Analysis of Attenuation Zones of 2D Periodic Foundations
}

\author{
Xinnan LIU ${ }^{\mathrm{a}}$, Yuan TIAN ${ }^{\mathrm{b}, 1}$, Yihe $\mathrm{WANG}^{\mathrm{c}}$, Yiqiang REN ${ }^{\mathrm{a}}$ and Xiaoruan SONG ${ }^{\text {a }}$ \\ ${ }^{a}$ School of Civil Engineering, North China University of Technology, Beijing 100144, \\ China \\ ${ }^{\mathrm{b}}$ Transportation Technology Development Promotion Center, China Academy of \\ Transportation Sciences, Beijing 100029, China \\ ${ }^{\mathrm{c}}$ Beijing Urban Construction Yatai Group Co. Ltd, Beijing 100013, China
}

\begin{abstract}
In this paper, global sensitivity analyses of attenuation zones of 2D periodic foundations are conducted. Global sensitivity analyses of upper bound frequency and lower bound frequency of the 1st attenuation zone of 2D periodic foundation are conducted considering four input parameters, i.e., initial stress ratio, filling ratio of core, filling ratio of resonator and periodic constant. Interactions and relative importance of input parameters are calculated.
\end{abstract}

Keywords. Periodic foundations, global sensitivity analysis, Sobol' decomposition, Gauss-Lobatto integration

\section{Introduction}

Periodic composite structures exhibit unique wave propagation property, i.e., attenuation zones (AZs) where elastic or acoustic waves are prohibited to propagate. The unique wave propagation property of periodic composite structures has also motivated researchers to design periodic foundations and seismic metamaterials to isolate the dominant frequency components of seismic waves [1-2].

In the previous studies of AZs of periodic foundations, the parametric analysis and sensitivity analysis are mainly conducted by varying only one parameter at a time with other parameters keeping unchanged [3-5]. However, this kind of parametric analysis is not able to recognize the most influential parameter or investigate the interactions of different parameters. In the present paper, the global sensitivity analysis method combined with the Gauss-Lobatto integration is extended to investigate the AZs of 2D periodic foundations. The rest of the present paper is organized as follows. In Section 2, the computational method is developed. Global sensitivity analyses of upper bound frequency (UBF), and lower bound frequency (LBF) of the 1 st AZ are performed in Section 3. In addition, fitting functions of the LBF and UBF of the 1st AZs are established in Section 3. Finally, some conclusions are drawn in Section 4.

1 Yuan Tian, Transportation Technology Development Promotion Center, China Academy of Transportation Sciences, Beijing 100029, China; E-mail: ytian88@163.com. 


\section{Governing equations}

\subsection{AZs of 2D Periodic Foundations}

Figure 1 illustrates the schematic of a 2D periodic foundation. In this paper, the effect of upper loads due to the superstructure was considered as uniformly distributed homogeneous initial stress $\sigma_{z z}^{0}$ in the $2 \mathrm{D}$ periodic foundation for simplicity. A typical unit cell can be identified, as shown in figure 1, where the thickness of coating layer is $t_{k}$, the square cylinder core length of a side is $l$ and the periodic constant i.e., the side length of the unit cell is $a$.

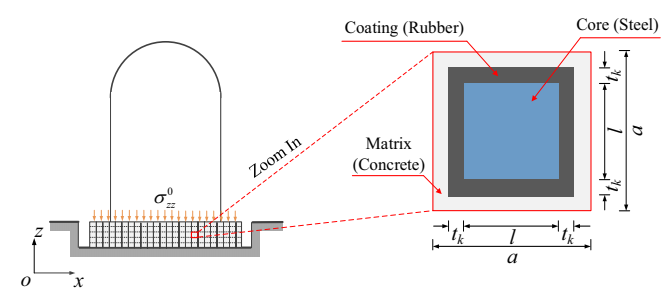

Figure 1. Schematic of a 2D periodic foundation.

The basic equations in an infinite homogeneous solid with initial stress due to the superstructure can be given as

$$
\sigma_{i j, j}+\left(u_{i, k} \sigma_{k j}^{0}\right)_{, j}=\rho \ddot{u_{i}}
$$

where $i, j, k=x, y, z, \sigma_{i j}$ is the stress tensor, the comma followed by the subscript $j$ denotes space differentiation with respect to the $j$ coordinate, $u_{i}$ is the displacement in the $i$ coordinate direction, $\sigma_{k j}^{0}$ is the initial stress tensor, $\rho$ is the mass density, the dot over displacement represents time differentiation.

Consider a harmonic in-plane wave (coupled wave by $P$ and $S V$ waves) with the angular frequency $\omega$ and wave vector $\mathbf{k}=k_{x} \mathbf{e}_{x}+k_{z} \mathbf{e}_{z}$, where $k_{x}, k_{z}, \mathbf{e}_{x}$ and $\mathbf{e}_{z}$ are the phase constants and unit wave vectors in the $x$ - and $z$-directions, respectively. Let $u$ and $w$ denote the displacement components in the $x$ - and $z$-directions, respectively. The field equations for the in-plane waves in the $2 \mathrm{D}$ periodic foundation can be expressed as

$$
\begin{aligned}
& \frac{\partial \sigma_{x x}^{(e)}}{\partial x}+\frac{\partial \tau_{x z}^{(e)}}{\partial z}+\frac{\partial^{2} u^{(e)}}{\partial z^{2}} \sigma_{z z}^{0}=\rho^{(e)} \ddot{u}^{(e)}, \\
& \frac{\partial \sigma_{z z}^{(e)}}{\partial z}+\frac{\partial \tau_{x z}^{(e)}}{\partial x}+\frac{\partial^{2} w^{(e)}}{\partial z^{2}} \sigma_{z z}^{0}=\rho^{(e)} \ddot{w}^{(e)},
\end{aligned}
$$

where the superscript $e$ denote the material of the cores, coating layers, and matrix.

The strains in the $2 \mathrm{D}$ periodic foundation can be given as 


$$
\varepsilon_{x}^{(e)}=\frac{\partial u^{(e)}}{\partial x}, \quad \varepsilon_{z}=\frac{\partial w^{(e)}}{\partial z}, \gamma_{x z}=\frac{\partial u^{(e)}}{\partial z}+\frac{\partial w^{(e)}}{\partial x}
$$

The constitutive equations can be expressed as

$$
\left[\begin{array}{c}
\sigma_{x x}^{(e)} \\
\sigma_{z z}^{(e)} \\
\tau_{x z}^{(e)}
\end{array}\right]=\frac{\bar{E}^{(e)}}{1-\bar{v}^{(e) 2}}\left[\begin{array}{ccc}
1 & \bar{v}^{(e)} & 0 \\
\bar{v}^{(e)} & 1 & 0 \\
0 & 0 & \left(1-\bar{v}^{(e)}\right) / 2
\end{array}\right]\left[\begin{array}{l}
\varepsilon_{x x}^{(e)} \\
\varepsilon_{z z}^{(e)} \\
\gamma_{x z}^{(e)}
\end{array}\right]
$$

where $\left\{\begin{array}{l}\bar{E}^{(e)}=\frac{E^{(e)}}{1-v^{(e) 2}} \\ \bar{\mu}^{(e)}=\frac{v^{(e)}}{1-v^{(e)}}, v^{(e)} \text { and } E^{(e)} \text { are the Poisson ratio and Young modulus of the }\end{array}\right.$ material $e$.

According to the Bloch-Floquet theory, the periodic boundary conditions of the typical unit cell have the form of

$$
\Psi(\mathbf{r}+\mathbf{a}, t)=\Psi(\mathbf{r}, t) \mathrm{e}^{\mathrm{ik} \cdot \mathbf{a}}
$$

where $\Psi$ denotes the displacement and stress field function, $\mathbf{r}$ is the space vector of a point on the boundaries of a typical unit cell, $\mathbf{a}=a\left(n_{x} \mathbf{e}_{x}+n_{z} \mathbf{e}_{z}\right), n_{x}$ and $n_{z}$ are integers, $\mathrm{i}=\sqrt{-1}$.

By various numerical methods, such as the FEM and the WFQEM, the dynamic equations for in-plane waves in the $2 \mathrm{D}$ periodic foundation can be obtained

$$
\left[\mathbf{K}(\mathbf{k})+\mathbf{K}^{0}(\mathbf{k})-\omega^{2}(\mathbf{k}) \mathbf{M}\right] \mathbf{X}=\mathbf{0}
$$

where $\mathbf{M}, \mathbf{K}^{0}(\mathbf{k})$ and $\mathbf{K}(\mathbf{k})$ are the mass matrix, geometric stiffness matrix due to the initial stress and stiffness matrix of the unit cell, respectively; $\mathbf{X}$ is the nodal displacement vector. For a given wave vector $\mathbf{k}$, the eigenfrequencies $\omega$ can be obtained by Equation (6). By varying the wave vector $\mathbf{k}$ in the first irreducible Brillouin zone, the attenuation zones can be obtained by identifying the gaps in adjacent dispersion curves.

\subsection{Sobol' Sensitivity Analyses}

By using the Gauss-Lobatto integration, the accuracy of Sobol' sensitivity analysis method has been improved comparing with the traditional one based on the widely used Monte Carlo integration method. The algorithm of the Sobol' sensitivity analysis method combined with the Gauss-Lobatto integration can be found in the previous paper of the authors [6]. 


\section{Results and Discussion}

In this paper, the square cores, matrix and coating layers were made of steel, concrete, and rubber, respectively; and the material properties are shown in table 1 . In addition to the periodic constant, three other parameters of the $2 \mathrm{D}$ periodic foundation were considered in the global sensitivity analysis, i.e., the filling ratio of core $f_{1}=\left(\frac{l}{l+2 t_{k}}\right)^{2}$, filling ratio of resonator $f_{2}=\left(\frac{l+2 t_{k}}{a}\right)^{2}$ and initial stress ratio $\alpha=\left|\frac{\sigma_{z z}^{0}}{G_{r}}\right|$, respectively, where $G_{r}$ is the shear modulus of the rubber layer. The reason of choosing shear modulus of the rubber layer as the reference of initial stress is that shear modulus of the rubber layer is the critical buckling stress of the $2 \mathrm{D}$ periodic foundation for out-ofplane waves.

Figure 2 shows the attenuation zones and dispersion curves with various parameters, where (a) $f_{1}=0.25, f_{2}=0.5, a=2 \mathrm{~m}$ and $\alpha=0$, (b) $f_{1}=0.25$, $f_{2}=0.5, a=1.5 \mathrm{~m}$ and $\alpha=0$, (c) $f_{1}=0.5, f_{2}=0.5, a=1.5 \mathrm{~m}$ and $\alpha=0$, and (d) $f_{1}=0.5, f_{2}=0.5, a=1.5 \mathrm{~m}$ and $\alpha=0.4$. Gaps between dispersion curves in figure 2 are the so-called band gaps or AZs, whose range is from the lower bound frequency (LBF) to upper bound frequency (UBF). It can be found that the bigger the side length of the unit cell is, the narrower and lower the 1 st $A Z$ is, by comparing figure 2(a) with figure 2(b). By comparing figure 2(b) with figure 2(c), it can be found that the larger the filling ratio of core is, the higher and wider the 1st AZ is. By comparing figure 2(c) with figure 2(d), it can be found that the initial stress shifts the 1 st AZ to lower frequency and narrows the 1 st $\mathrm{AZ}$.

The above comparing process is the typical parametric analysis process, where only one parameter is changed at a time with the rest of parameters keeping unchanged. Thus, it cannot manage to investigate the interactions and relative importance of the parameters in determining the 1 st $\mathrm{AZ}$.

Table 1. Material parameters

\begin{tabular}{cccc}
\hline Materials & Mass density & Poisson ratio & Young modulus \\
& $\rho\left(\mathrm{kg} / \mathrm{m}^{3}\right)$ & $v$ & $E(\mathrm{GPa})$ \\
\hline \multirow{2}{*}{ Steel } & 1300 & 0.463 & $1.37 \times 10^{-4}$ \\
Concrete & 7850 & 0.330 & 210 \\
& 2300 & 25 & 0.330 \\
\hline
\end{tabular}

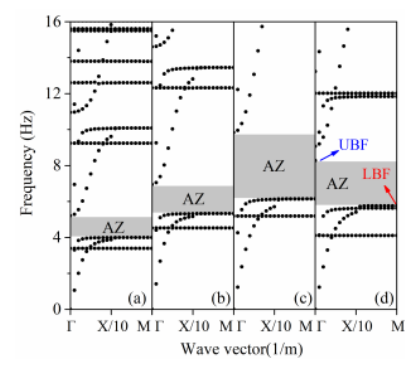

Figure 2. Dispersion curves and attenuation zones. 


\subsection{Global Sensitivity Analyses of the 1st AZ}

Four parameters are used in the global sensitivity analyses, as listed in table 2 . The ranges of these parameters are set so that the most acceptable size and initial stress of foundations in practice can be included. Two response functions of the LBF and UBF were used to characterize the 1 st AZ.

The 1st two order Sobol' indices of the LBF are calculated by different numbers of grid points, as shown in figure 3. It can be seen from figure 3 that the Sobol' indices of LBF converge for $N=5$, which verifies that the convergence of the present method is fast. Figure 4 shows Sobol' indices of the LBF of the 1st AZ by ranking. Moreover, the accumulated values of Sobol' indices are calculated. From figure 4, it can be easily found that the filling ratio of resonator is the most important parameter in determining the LBF of the 1st AZ while the periodic constant and filling ratio of core have relatively moderate importance. Moreover, the interactions of the four input parameters are negligible while the sum of the first order Sobol' indices accounts for $98.9 \%$ of the total variance.

Figure 5 shows the 1st two order Sobol' indices of the UBF calculated by different numbers of grid points. Figure 6 shows accumulated values in sequence and Sobol' indices of the UBF by ranking. From figure 6, it can be found that the filling ratio of core is the most important factor in determining the UBF of the 1st AZ, which is followed by the periodic constant, filling ratio of resonator and initial stress ratio. Moreover, the interactions of the four input parameters almost have no effects on the UBF of the 1st AZ.

Table 2. Parameters used in the global sensitivity analyses

\begin{tabular}{lll}
\hline Parameters & Range of parameters & Sobol' index \\
\hline Filling ratio of core $\left(f_{1}=\left(\frac{l}{l+2 t_{k}}\right)^{2}\right)$ & {$[0.2,0.6]$} & $S_{1}$ \\
\hline Filling ratio of resonator $\left(f_{2}=\left(\frac{l+2 t_{k}}{a}\right)^{2}\right)$ & {$[0.2,0.6]$} & $S_{2}$ \\
\hline Periodic constant $(a)$ & {$[1.5,2.0] \mathrm{m}$} & $S_{3}$ \\
\hline Initial stress ratio $\left(\alpha=\left|\frac{\sigma_{z z}^{0}}{G_{r}}\right|\right)$ & {$[0,0.8]$} & $S_{4}$ \\
\hline
\end{tabular}

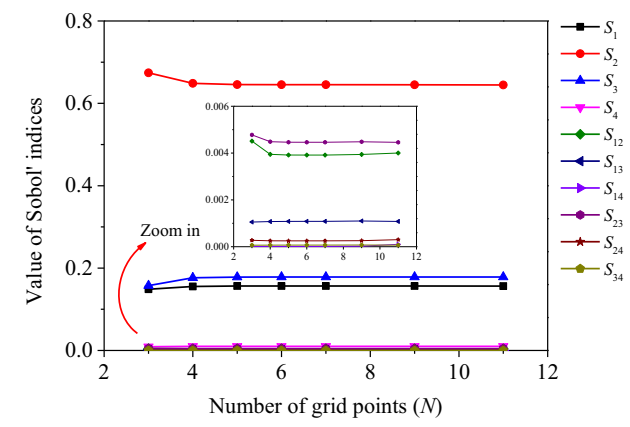

Figure 3. 1st two order Sobol' indices for the LBF. 


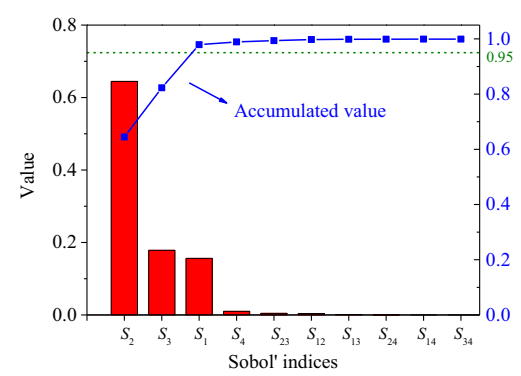

Figure 4. Ranking of Sobol' indices for the LBF.

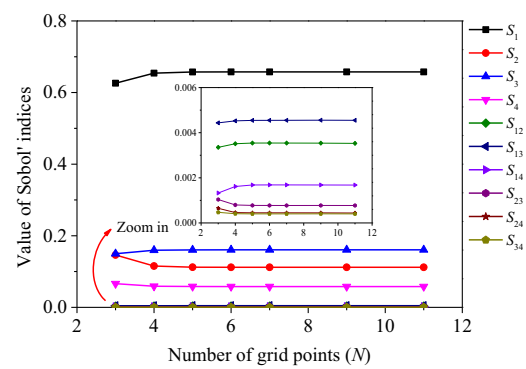

Figure 5. 1st two order Sobol' indices of the UBF.

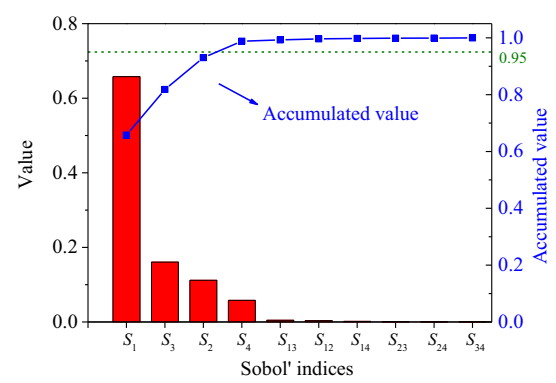

Figure 6. Ranking of Sobol' indices of the UBF.

\subsection{Fitting Equations}

Sobol' functions $F_{0}, F_{1}, F_{2}, F_{3}$ and $F_{4}$ are used to estimate the LBF of the 1st AZ, which leads to $98.9 \%$ confidence according to figure 4 . Figure 7 shows the Sobol' functions $F_{1}, F_{2}, F_{3}$ and $F_{4}$ and their corresponding fitting curves. From figure 7(a), it can be found that $F_{1}$ increases as the filling ratio of core increases. In figures 7(b), (c) and (d), monotonically decreasing trend of $F_{2}, F_{3}$ and $F_{4}$ can be observed, respectively. The fitting function of the LBF of the 1st AZ has the form of

$$
F^{L B F} \approx F_{0}^{L B F}+F_{1}^{L B F}+F_{2}^{L B F}+F_{3}^{L B F}+F_{4}^{L B F}
$$

where 


$$
\begin{aligned}
& F_{0}^{L B F}=5.6273 \\
& F_{1}^{L B F}=3.9946 f_{1}^{3}+2.6335 f_{1}^{2}-0.4037 f_{1}-0.6139 \\
& F_{2}^{L B F}=-35.3560 f_{2}^{3}+58.1568 f_{2}^{2}-36.2449 f_{2}+7.2473 . \\
& F_{3}^{L B F}=1.8640 a^{2}-9.7691 a+11.3487 \\
& F_{4}^{L B F}=-0.1331 \alpha^{2}-0.3666 \alpha+0.1753
\end{aligned}
$$

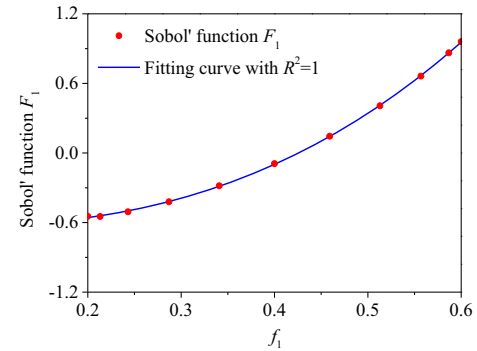

(a)

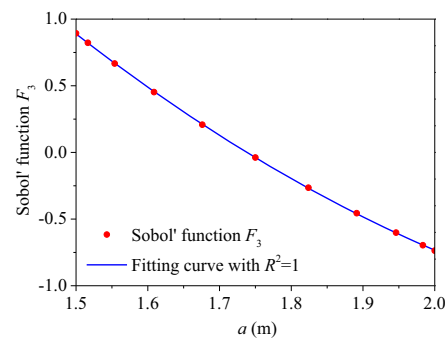

(c)

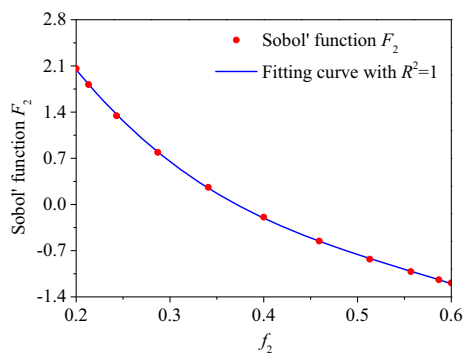

(b)

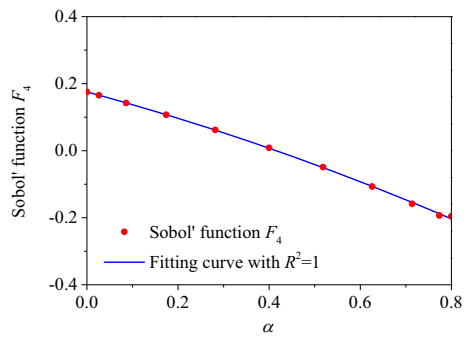

(d)

Figure 7. Sobol' functions (a) $F_{1}$, (b) $F_{2}$, (c) $F_{3}$ and (d) $F_{4}$ of the LBF of the 1 st AZ.

$F_{0}, F_{1}, F_{2}, F_{3}$ and $F_{4}$ are used to approximate the UBF of the 1 st AZ, which leads to $98.8 \%$ confidence according to figure 6 . The fitting function of the UBF of the 1 st $\mathrm{AZ}$ can be expressed as

$$
F^{U B F} \approx F_{0}^{U B F}+F_{1}^{U B F}+F_{2}^{U B F}+F_{3}^{U B F}+F_{4}^{U B F}
$$

where

$$
\begin{aligned}
& F_{0}^{U B F}=7.3903 \\
& F_{1}^{U B F}=11.7840 f_{1}^{2}+1.3500 f_{1}-2.5819 \\
& F_{2}^{U B F}=-32.9644 f_{2}^{3}+54.9942 f_{2}^{2}-31.5384 f_{2}+5.7215 . \\
& F_{3}^{U B F}=2.4479 a^{2}-12.8296 a+14.9041 \\
& F_{4}^{U B F}=-2.068 \alpha^{3}+0.8433 \alpha^{2}-1.0486 \alpha+0.5036
\end{aligned}
$$




\section{Conclusions}

The global sensitivity analyses of the AZs of 2D periodic foundations is conducted. It should be noted that the proposed method is general and applicable to various types of sensitivity analysis problems in a similar way. By investigating global sensitivity of the upper bound frequency and lower bound frequency of the 1 st AZ considering four input parameters within the range in table 2 , the following conclusions can be drawn:

1) The Gauss-Lobatto integration converges fast, which makes it a promising global sensitivity analysis method due to its higher accuracy and lower computational cost.

2) The filling ratio of resonator is the most important parameter in determining the LBF of the 1st AZ while the periodic constant and filling ratio of core have relatively moderate importance. The filling ratio of core is the most important parameter in determining the UBF of the $1 \mathrm{st} \mathrm{AZ}$, which is followed by the periodic constant, filling ratio of resonator and initial stress ratio.

3) The interactions of the initial stress ratio, filling ratio of core, filling ratio of resonator and periodic constant and can be negligible in determining the 1st AZs of 2D periodic foundations.

\section{Acknowledgment}

This work is supported by the National Natural Science Foundation of China (51808007).

\section{References}

[1] Xiang HJ, Shi ZF, Wang SJ, Mo YL. Periodic materials-based vibration attenuation in layered foundations: Experimental validation. Smart Mater. Struct. 2012 Nov; 21; 112003.

[2] Basone F, Wenzel M, Bursi OS, Fossetti M. Finite locally resonant Metafoundations for the seismic protection of fuel storage tanks. Earthq. Eng. Struct. D. 2019 Feb; 48: 232-52.

[3] Cheng ZB, Shi ZF. Composite periodic foundation and its application for seismic isolation. Earthq. Eng. Struct. D. 2018 Apr; 47: 925-44.

[4] Meng QJ and Shi ZF. Propagation attenuation of plane waves in single-phased soil by periodic pile barriers. Int. J. Geomech. 2018 Jun; 18; 04018035.

[5] Witarto W, Nakshatrala KB and Mo YL. Global sensitivity analysis of frequency band gaps in onedimensional phononic crystals. Mec. Mater. 2019 Jul; 134: 38-53.

[6] Liu XN, Ren YQ, Song XR and Witarto W. A global sensitivity analysis method based on the GaussLobatto integration and its application in layered periodic foundations with initial stress. Compos. Struct. 2020 Jul; 244; 112297. 